\title{
REVIEW \\ Polyploidy and its effect on evolutionary success: old questions revisited with new tools
}

\begin{abstract}
A Madlung
Polyploidy, the condition of possessing more than two complete genomes in a cell, has intrigued biologists for almost a century. Polyploidy is found in many plants and some animal species and today we know that polyploidy has had a role in the evolution of all angiosperms. Despite its widespread occurrence, the direct effect of polyploidy on evolutionary success of a species is still largely unknown. Over the years many attractive hypotheses have been proposed in an attempt to assign functionality to the increased content of a duplicated genome. Among these hypotheses are the proposal that genome doubling confers distinct advantages to a polyploid and that these advantages allow polyploids to thrive in environments that pose challenges to the polyploid's diploid progenitors. This article revisits these long-standing questions and explores how the integration of recent genomic developments with ecological, physiological and evolutionary perspectives has contributed to addressing unresolved problems about the role of polyploidy. Although unsatisfactory, the current conclusion has to be that despite significant progress, there still isn't enough information to unequivocally answer many unresolved questions about cause and effect of polyploidy on evolutionary success of a species. There is, however, reason to believe that the increasingly integrative approaches discussed here should allow us in the future to make more direct connections between the effects of polyploidy on the genome and the responses this condition elicits from the organism living in its natural environment.
\end{abstract}

Heredity (2013) 110, 99-104; doi:10.1038/hdy.2012.79; published online 14 November 2012

Keywords: polyploidy; polyploidization; speciation; evolution; stress

\section{INTRODUCTION}

Polyploids are organisms whose genomes consist of more than two complete sets of chromosomes. Stebbins distinguished three major types of polyploids: autopolyploids, allopolyploids and segmental allopolyploids (Stebbins, 1947). In autopolyploids all genomes are identical or very similar and arise via genome duplication within the same species (Stebbins, 1947; Lewis, 1980). Allopolyploids, by contrast, contain two or more distinct genomes, and can arise via hybridization of two different species concomitant with genome doubling (Stebbins, 1947; Grant, 1975). Segmental allopolyploids carry more than two partially differentiated genomes, which can lead to the formation of both bivalents and multivalents during chromosome pairing (Stebbins, 1947; Levin, 2002).

Major routes of polyploid formation are via gametic nonreduction and, to a lesser degree, somatic doubling (Lewis, 1980; Ramsey and Schemske, 1998; Levin, 2002; Coyne and Orr, 2004). In gametic non-reduction, fusion of two gametes, of which at least one contains a non-reduced, full somatic complement of chromosomes, can lead to polyploidy. Somatic doubling may occur in zygotic, embryonic or sporophytic tissue. Spontaneous genome duplication in those tissues can thus also produce viable polyploid offspring via gamete formation in the duplicated sectors (Ramsey and Schemske, 1998).

Polyploidization in general is now believed to have occurred at least once during the evolutionary history of all angiosperms (Jiao et al., 2011) and it has been suggested as a major driving force of plant evolution (Blanc and Wolfe, 2004; Chen, 2007; Soltis and Soltis,
2009). Indeed, polyploidization has been described as a process leading to instantaneous speciation (Mayr, 1963) because a singlegeneration event such as the hybridization between two species with subsequent somatic doubling, or the fusion of unreduced gametes is enough to quickly establish barriers that prevent gene flow between the new polyploid and the old progenitor species, and can lead to reproductive isolation (Ramsey and Schemske, 1998).

Although auto- and allopolyploidy share the property of duplicated genomes, the difference in their compositions has important consequences. In autopolyploids, chromosomes generally pair as multivalents during meiosis, while in allopolyploids bivalent pairing between chromosomes of the same original genome is prevalent (Stebbins, 1947), resulting largely in the maintenance of two separate genomes. However, the more closely genomes in the allopolyploid are related, the more likely it is for homoeologs (the chromosomes duplicated by allopolyploidy) to pair (Levin, 2002), resulting in chromosomal exchanges between the two genomes.

Despite the interest in polyploidy there are many long-standing, yet still unanswered questions about the significance of genome doubling, especially in the areas of ecology, physiology and evolution (Soltis et al., 2010). In this review, I revisit two of these questions and discuss how recent genomic developments have furthered the discussion on these questions. I aim to pay special attention to areas where complementary approaches of different disciplines are starting to be synergistic and where continued integration of genomic with ecological and organismal information would help in the future to resolve unanswered problems. 


\section{IS POLYPLOIDY ADVANTAGEOUS FOR EVOLUTIONARY SUCCESS?}

There has been a long-standing debate over the evolutionary implications of genome doubling (Winge, 1917; Müntzing, 1936; Ohno, 1970; Comai, 2005; Otto, 2007; Soltis and Soltis, 2009), which span the spectrum from polyploidy being an evolutionary dead end (Stebbins, 1950; Arrigo and Barker, 2012) to polyploidy being a mayor player with a significant role in evolution (Ohno, 1970; Chen, 2010; Mayfield et al., 2011).

Three major advantages are often cited that should give polyploids an edge over their diploid parents. First, the increased number of alleles of a given gene in a polyploid should allow the masking of deleterious recessive mutations and thus insure against the loss of fitness ( $\mathrm{Gu}$ et al., 2003). The second proposed advantage of allopolyploids and heterozygous autopolyploids is that heterosis allows offspring to display transgressive performance compared with its progenitor species (Birchler et al., 2010). In contrast to diploid hybrids where hybrid vigor decays over subsequent generations due to homologous recombination, heterosis is stable in allopolyploids due to the predominant disomic pairing of identical homologous chromosomes (Ohno, 1970; Comai, 2005). The third major advantage of polyploids stems from the possibility that duplicated gene copies can evolve to assume new or slightly varied functions (neofunctionalization or subfunctionalization), potentially allowing for ecological niche expansion or increased flexibility in the organism's responsiveness to environmental change (Adams and Wendel, 2005; Moore and Purugganan, 2005; Lynch, 2007).

Among the disadvantages that could lead to less vigor and a reduced adaptive capacity in polyploids are the increased number of chromosomes, and the greater complexity of their pairing and segregation interactions that can cause abnormalities (including aneuploidy) during meiosis and mitosis (Comai, 2005). Additionally, the cell architecture in polyploids is altered because of generally increased cell size in polyploids, which alters the surface to volume ratio (Speckman et al., 1965; Rhoades and Dempsey, 1966; Melaragno et al., 1993; Levin, 2002). Of particular concern to newly formed outcrossing polyploids is the minority cytotype disadvantage. As pollination is more likely to occur with haploid pollen from neighboring diploid progenitor individuals than with diploid pollen from another polyploid, the major proportion of the offspring is likely to be triploid. Triploid individuals are then less likely to be viable, or if they are viable, they are less likely to be fertile (Levin, 1975). Finally, changes in polyploids that can be either advantageous or detrimental are the altered transcriptome, genomic architecture and epigenetic landscape, which can lead to gene silencing or activation, as well as DNA loss and epigenetic changes (Wang et al., 2004, 2006; Hegarty et al., 2006; Gaeta et al., 2007, 2009; Chaudhary et al., 2009; Pumphrey et al., 2009; Rapp et al., 2009; Wright et al., 2009; Buggs et al., 2010, 2011; Hegarty et al., 2011; Mayfield et al., 2011; Xiong et al., 2011; Matsushita et al., 2012). In the new polyploid such changes can lead to reduced, additive or transgressive states. Therefore, polyploidization produces offspring that has the potential to be distinctly different from both of its parents.

Despite the shortage of convincing evidence linking genomic change to physiologically based enhancement of traits and proven evolutionary success (as opposed to just the theoretical promise of evolutionary success), many of the recent articles cited above have concluded that polyploidy has a definitive role in evolution, and questioned or ignored Stebbins's notion that polyploidy might be an evolutionary dead end (Stebbins, 1950). Recently, investigators using modern phylogenetic approaches have revived the debate over the question to what degree polyploidy has a creative role in evolution (Wood et al., 2009; Mayrose et al., 2011; Arrigo and Barker, 2012). Wood et al. (2009) reported that while $15 \%$ of all speciation events in angiosperms coincided with an increase in ploidy, subsequent faster speciation rates-as might be expected if the duplicated genome indeed allowed for the new polyploid species to populate new ecological niches or have a broader response to environmental change-did not seem to be the norm for these species (Wood et al., 2009). Using a comprehensive phylogenetic data set and estimating rates of diversification in newly formed polyploids, Mayrose et al. (2011) determined that polyploids were more likely to be found at the branch tips of phylogenetic trees, and concluded that polyploidy was often followed by extinction as opposed to diversification. Their analyses further showed that speciation rates of polyploid plants were on average lower than those in diploid plants (Mayrose et al., 2011), suggesting that polyploidy is actually more likely to be detrimental than advantageous for evolutionary success. From these two studies one might infer (a) that polyploidy is a condition that occurs frequently and accidentally but does not generally lead to further speciation events after polyploidization, (b) that evolutionary success of neopolyploids is possible but not the norm and (c) that extinction of recently formed species occurs more frequently in polyploids than in diploid relatives (Arrigo and Barker, 2012). On the other hand, Mayrose et al. (2011) also suggested that increased genomic diversity in neopolyploids could be the key to success in those new polyploids that did persevere during the establishment of the new species (Mayrose et al., 2011).

In considering the implications of the two phylogenetic studies described in the previous paragraph (Wood et al., 2009; Mayrose et al., 2011), it is important to note that neither study specifically distinguished between auto- and allopolyploidy. These two conditions can be difficult to distinguish phylogenetically but the differences between allopolyploidy versus auto-polyploidy can have significant and different consequences on the organism (Grant, 1971; Levin, 2002), and thus likely its potential for evolutionary success. If autoand allopolyploids indeed have different rates of success in evolution, it is important to study their responses to polyploidy separately if the question is whether genome doubling is advantageous or disadvantageous. While it is not trivial to distinguish between the effects of hybridization and genome doubling in allopolyploidization events, distinct differences between genome doubling and hybrid effects were described in several recent studies in Arabidopsis, Senecio (ragwort) and Gossypium (cotton). In Arabidopsis, Wang et al. (2006) used microarrays to measure transcriptome changes in neoallopolyploids compared with their autotetraploid parents and found hundreds of genes to be differentially regulated between parents and offspring. The same study, using autopolyploids that had gone through wholegenome duplication but not through hybridization, displayed considerably fewer changes between tetraploids and diploid progenitors. This led the authors to suggest that hybridization, not genome duplication, was the driving force in transcriptomic change in allopolyploids (Wang et al., 2006; Chen, 2007). Two subsequent studies that analyzed transcriptomic changes in Arabidopsis autopolyploids only partially supported the view that hybridization resulted in greater genomic change than genome doubling. Pignatta et al. (2010) used three independently produced sets of autopolyploids and diploids that arose from differently affected sectors of colchicinetreated parent Arabidopsis (ecotype Landsberg erecta, Ler) individuals. While they found no genes that were consistently differentially regulated in response to genome doubling in their microarray analysis, they did observe significant differences between transgenic 
diploids and tetraploids carrying tissue-specific green fluorescent protein genes activated by endogenous enhancer elements ('enhancer traps'). Differential expression of these enhancers between diploids and tetraploids suggested that perhaps a less complex and more ploidy-sensitive regulation of enhancer traps compared with native genes was responsible for this difference (Pignatta et al., 2010). Yu et al. (2010) reported that the magnitude of transcriptomic changes in response to genome doubling in diploid and autotetraploid Arabidopsis was dependent on the ecotype used. These authors reported several hundred differentially expressed genes in seedlings or leaves in the ecotype Columbia, but only 9 and 22 genes that were differentially expressed in seedlings and leaves, respectively, in the ecotype Ler (Yu et al., 2010), the same ecotype that Wang et al. (2006) had used in their study of transcriptomic changes in auto- and allopolyploids.

Using the Senecio system, Hegarty et al. $(2005,2006)$ studied the separate effects of hybridization and genome duplication by first comparing the synthetic triploid hybrid Senecio $x$ baxteri with its two progentior species, the diploid $S$. squalidus and the tetraploid $S$. vulgaris. Subsequently, genome doubling was induced in the triploid using colchicine (Hegarty et al., 2005, 2006) to generate hexaploids equivalent to the naturally occurring $S$. cambrensis. Microarray-based transcriptome analysis in this system suggested that both hybridization and genome doubling had profound effects on the transcriptome but that these effects were distinct from each other (Hegarty et al., 2005, 2006). Furthermore, the authors reported that genome doubling reduced the number of differentially regulated genes in the hybrid compared with the number of differentially regulated genes in the allohexaploid (when either one was compared with their progenitor species), and suggested that genome doubling, therefore, has a 'calming effect' on the hybridization-induced transcriptome shock (Hegarty et al., 2006).

To distinguish between the effects of hybridization and allopolyploidization, Flagel et al. (2008) compared gene expression changes between a synthetically produced F1 hybrid and those in a natural allopolyploid of the same original parentage in cotton (Gossypium). These authors reported that around $24 \%$ of genes with a significantly differential regulation in the F1 hybrid were also found to have the same expression bias in the natural allopolyploid when compared with gene expression in the parents, suggesting that these genes were altered due to genome merger (Flagel et al., 2008). The remaining $76 \%$ of expression changes that were observed in the natural allopolyploid (compared with its progenitor species) could reflect accumulated mutations and sub- or neofunctionalized duplicate genes that had assumed altered expression patterns over evolutionary time in the polyploid (Flagel et al., 2008). Although the experimental design of this study did not allow the authors to make direct inferences of the effect of genome doubling on changes in gene expression, they were able to conclude that genome merger had a significant but likely only partial role in altering expression patterns between diploids and hybrids or allopolyploids (Flagel et al., 2008).

From the discussion of the studies cited above (Hegarty et al., 2005, 2006; Wang et al., 2006; Flagel et al., 2008; Pignatta et al., 2010; Yu et al., 2010) it appears that different species, and even different ecotypes of the same species, can vary significantly in their responses to hybridization, genome doubling or the combination of the two. If we assume that the differential expression of the genome in all polyploids compared with their parents has a role in the evolutionary trajectory of the neopolyploids, then it is tempting to speculate that the magnitude of transcriptomic change in the neopolyploid (or the specific change of transcriptional activity in some key genes) can have a deciding role over which polyploids will persevere in evolution, and for which polyploids genome duplication leads to a dead end. A similarly important aspect that warrants more investigation is the question to what degree the genomic changes that occur during polyploidization lead to increased variation in the sibling offspring. Whether or not polyploidy induces greater variation between siblings than is found in diploid populations is still an open question (Lee and Chen, 2001; Gaeta et al., 2007; Madlung et al., 2012). It is important to realize that despite the increased variation-and thus evolutionary potential-described in many of the genomic studies discussed in this article, survival or vigor of neopolyploids in the Arabidopsis, Brassica and corn study systems have often been low in synthetic populations (Comai et al., 2000; Madlung et al., 2005; Riddle et al., 2006; Gaeta et al., 2007; Matsushita et al., 2012), suggesting either a severe bottleneck for the establishment of vigorous polyploids (Comai, 2005), or the beginning of extinction. Likewise, and very importantly, we still cannot infer from the integration of the molecular and phylogenetic studies discussed earlier whether there is a difference in polyploid advantage or disadvantage for autopolyploids compared with allopolyploids. Molecular analysis of more species may help determine if significant transcriptome changes in response to genome duplication are the rule or the exception as seen in the example of ecotype variation in Arabidopsis (Wang et al., 2006; Yu et al., 2010). And finally, to determine if polyploidy-induced genomic changes are adaptive, we also need more integrative studies assessing the physiological and ecological consequences of the genomic changes.

Despite the relative rarity of radiation associated with polyploid speciation discussed earlier (Wood et al., 2009; Mayrose et al., 2011; Arrigo and Barker, 2012), it is important to recognize the evolutionary potential of polyploidy. For that purpose, I will briefly discuss two individual examples of older evolutionarily successful polyploids, which not only survived polyploidization, but for which genome doubling were followed by additional speciation events. Since its split from a common ancestor, Gossypium has evolved into three distinct lineages throughout the world: the New World clade (or D-genome); the African-Asian clade separated into the A-, B-, E- and F-genomes; and the Australian clade with the C-, G- and K-genomes (Wendel and Cronn, 2003). Ancestral lines of Gossypium are diploid, but an allopolyploidization event took place probably between 1-2 mya after long-distance dispersal of the A-genome to the New World, where it hybridized with the D-genome (Wendel and Cronn, 2003). This new allopolyploid has since radiated into five separate, morphologically diverse species: G. mustelinum, G. darwinii, G. barbadense, G. tomentosum, and the most important global supplier of agriculturally used cotton fiber G. hirsutm (Fryxell, 1979; Wendel and Cronn, 2003). A second example of an older allopolyploid that has successfully diversified is the genus Glycine (soybean). Glycine is a complex genus that underwent a whole-genome duplication an estimated 15 mya (Schlueter et al., 2004) and multiple fairly recent allopolyploidization events after its colonization of the Australian continent, for example, with the formation of G. tabacina about $30 \mathrm{~K}$ years ago (Doyle et al., 1999). In another polyploid species in the genus Glycine, G. tomentella, multiple separate 'races' are recognized that have not yet taxonomically arisen to species status, but that display enough molecular phylogenetic divergence from one another to be grouped into separate taxa (Doyle et al., 2004). These two examples from cotton and soybean suggest that diversity and speciation may indeed be found in nature in relatively recent polyploids, exemplifying how molecular diversity induced by polyploidization might have been the driving force in these speciation events. These two case studies are consistent with the possibility that polyploidization in some species can lead to subsequent diversification, when genome doubling in 
many other species was followed by extinction (Arrigo and Barker, 2012). Nonetheless, it is also important in this context to note that the coincidence of polyploidy and subsequent speciation does not preclude other mechanisms that may have had a role in the evolutionary success of cotton and soybean, such as gene flow between populations, recurrent polyploid origins and hybridization or introgression between different species after polyploidization (Coyne and Orr, 2004).

\section{DO POLYPLOIDS HAVE A GREATER POTENTIAL FOR ADAPTATION TO VARIABLE OR STRESSFUL ENVIRONMENTS?}

In the context of evolutionary success of polyploidy the question whether or not polyploids can adapt better to stressful environments was raised early on and has been studied extensively, and discussed at times controversially, in the older literature (Hagerup, 1932; Müntzing, 1936; Gustavsson, 1946; Löve and Löve, 1949; Stebbins, 1950; Löve, 1953; Grant, 1971). Polyploids have been reported to be more frequent in extreme environments, including the subarctic regions, high elevations and xeric environments (Löve and Löve, 1949; Löve, 1953; Hanelt, 1966; Grant, 1971). Given the hypothesis that polyploids can thrive relative to their diploid progenitors, polyploids have been studied for their morphological, physiological and developmental differences from diploids to find correlative evidence that might explain observations of higher stress endurance. One example of morphological differences between diploids and polyploids are the larger cell sizes in polyploids (Melaragno et al., 1993) including those of the stomata (Speckman et al., 1965; Masterson, 1994; Hodgson et al., 2010). Changes in stomatal pore size in response to polyploidy could suggest an effect of genome duplication on the water relations of the plant. A lower density of stomata in polyploids compared with diploids as found in Betula payrifera (birch) (Li et al., 1996) could on the other hand reduce or offset any differences in the overall gas exchange rates. Additionally, morphological variation in leaf thickness, pubescence, cuticular thickness or -composition, as well as physiological parameters affecting the plant's water potential can also have a role in water relations and gas exchange rates (Johnson, 1975; Li et al., 1996).

Integrative and experimental approaches to questions regarding the adaptive role of polyploidy in responses to the environment are still rare. Maherali et al. (2009) compared natural diploid, tetraploid and colchicine-induced neotetraploids of fireweed (Chamerion angustifolium) to study the effect of genome doubling on water relations. The authors reported larger stomata, increased stem and vessel diameter, and decreased specific hydraulic conductivity in both types of tetraploids over the diploids, but noted that the established tetraploids showed significantly greater drought tolerance than the similarly responding diploid and neotetraploid, suggesting that drought tolerance in fireweed likely evolved after and independently of genome duplication (Maherali et al., 2009). Ramsey (2011) used transplant experiments in the field to test survivorship of seedlings raised in the greenhouse and transplanted into a dry dune habitat. $\mathrm{He}$ reported a fivefold greater survivorship of seedlings raised from field collected seeds of hexaploids over tetraploids, and a 70\% greater survivorship of seedlings raised from seeds of spontaneous neohexaploids compared with tetraploids (Ramsey, 2011). Ramsey concluded from these experiments that polyploidy per se can have a role in adaptation to a new environment (Ramsey, 2011).

In another recent study with an integrative experimental design examining the ecophysiological implications of an altered morphology in polyploids, Li et al. (2012) examined the underlying mechanisms of cell size variation in diploid and tetraploid Arabidopsis thaliana with molecular tools. Using quantitative PCR, these authors queried expression differences in 34 cell cycle regulating genes and reported significant differences in the expression of three inhibitors of cyclin dependent kinases (ICK1, 2, 5) between diploids and tetraploids. These results suggest a mechanism by which altered gene expression levels of select genes could have direct results on the morphology and ecology of the plant. The results by Li et al. (2012) set the stage for follow-up experiments that could, for example, test if experimentally altered gene expression of ICK 1,2 and 5 has direct or proportional consequences on stoma size and if so, if variable stoma sizes have proportional effects on the water relations of the plant. It would also be of great interest if the described changes in transcriptional activity are the same in different populations of polyploids derived from separate polyploidization events, or if they are stochastic changes. If changes in gene expression levels of specific genes in multiple separately induced polyploids could directly be linked to physiological or morphological changes in polyploids compared with their parents, this would indicate that genes exist, which are directly and consistently regulated by genome doubling. In this context, it is interesting that recent genome wide studies using microarray technology in Arabidopsis (Wang et al., 2006; Pignatta et al., 2010; Yu et al., 2010), maize (Riddle et al., 2010), and potato (Stupar et al., 2007) have reported numerous transcriptional changes between ploidy levels, but did not uncover genes that were specifically ploidy-regulated or could have easily explained morphological differences between genotypes of different ploidy levels. Li et al. (2012) argue that maybe subtle changes below the detection limit of the array-based experiments, yet discernable by quantitative PCR, could contribute significantly to the observed morphological variation.

In contrast to the studies on autopolyploids cited in the previous section, recent molecular approaches from several labs have shown that allopolyploids display significant variation in gene expression compared with that of their parents (Adams et al., 2003; Wang et al., 2006; Flagel et al., 2008; Chaudhary et al., 2009; Buggs et al., 2010; Chelaifa et al., 2010), but in many cases unequivocal connections between the change in gene expression and an adaptive role in stress responses for the observed transcriptional change have not yet been made. A pair of recent studies in Coffea (coffee) addressed the question whether an allopolyploid compared with its parent species had a broader range of transcriptional expression patterns, and thus theoretically increased its chances to be better at responding to changing environmental conditions (Bardil et al., 2011; Combes et al., 2012). In the first of the two studies, the authors used microarray analysis to compare gene expression in Coffea arabica and its two progenitor species $C$. eugenioides and C. canephora. Their analysis showed that homoeologous gene pairs were differentially regulated in response to temperature in C. arabica (Bardil et al., 2011). In a separate study, the authors analyzed the relative contribution of each gene in homoeologous gene pairs to the overall expression of the gene in the allopolyploid and reported significant differences in their relative contribution to the overall transcription of the pair, depending on both tissue type and environmental conditions (Combes et al., 2012). This study suggested that the allopolyploid has a different, but not necessarily broader, range of responses to changing conditions. It would be interesting to see if the difference in expression could lead to a selective advantage, for example, in a comparative study of wholeplant performance of different individuals in different environments.

Homoeologous genes in the allopolyploid Gossypium hirsutum showed differential expression levels of each homoeolog when a variety of abiotic stresses, such as heat or cold, were applied (Liu and Adams, 2007; Dong and Adams, 2011). Also, in the allopolyploid 
Brassica napus, stressful environmental conditions led to a change in the identity of splice variants in a significant subset of homoeologous genes when compared to the two parent species $B$. olaracea and B. rapa (Zhou et al., 2011). Alternative splicing creates multiple mature mRNAs from a single gene, which can give rise to multiple proteins with different functions. Collectively, these studies provide strong experimental evidence that regulation of gene expression in homoeologs of allopolyploids can be variable in different conditions. Whether such changes in homoeolog regulation are adaptive, again, remains to be shown.

Another example for changed responsiveness to stress of allopolyploids relative to its parents comes from Arabidopsis. Here, mRNA stability was compared between allopolyploids and their parent species (Kim and Chen, 2011). Results from this study showed that genes involved in abiotic and biotic stress responses were significantly more likely to also have a differential mRNA decay time when comparing allopolyploids and progenitor species (Wang et al., 2006; Kim and Chen, 2011). These studies suggest that differential posttranscriptional regulation between allopolyploids and their progenitors might modulate the stress response of allopolyploids relative to their parent species and potentially allow survival under altered conditions.

Taken together, modern molecular studies suggest that the duplicated homoeologous genes in allopolyploids might be undergoing subfunctionalization, allowing the allopolyploid to differentially use the homoeologs for different or variable responses to an array of stressful conditions. This in turn would support the idea that allopolyploidization can lead to a functional increase in response options to a wider spectrum of potentially stressful environmental conditions, which could be evolutionarily adaptive. It is important to note, however, that in many cases we still lack direct evidence that polyploidy-induced variation is necessarily linked to the evolutionary potential of populations of polyploids. To gain answers to the question whether or not polyploidy promotes evolution via increased adaptability, more work is needed that integrates molecular and ecological approaches to test the role of specific genes on adaptation under field or controlled conditions.

\section{CONCLUDING REMARKS}

Despite the widespread occurrence of polyploidy in nature and the prevalence of its footprint in all angiosperm genomes, the question to what degree polyploidy affects the evolutionary trajectory of a species is still unclear. Old questions about the role of polyploidy in the response to stress or whether genome duplication is beneficial or detrimental to evolutionary success are being revisited using modern genomic tools.

Molecular studies have provided evidence for genomic change on numerous levels of regulation concomitant to polyploidization. However, in many cases we still don't know the effect of polyploidy on fitness under different environmental conditions, and there still is little evidence that observed transcriptional and genomic changes actually lead to faster evolution or greater adaptation in natural populations. Moreover, recent phylogenetic studies perhaps point to the contrary. It is possible that inherent differences in the types of responses to polyploidization are either species-specific or stochastic in individuals. Such differences may be the reason for why some polyploids, such as cotton and soybean, have been so successful and why, as described by Mayrose et al. (2011), so many others are not. To answer the question of the role of polyploidy per se on speciation, it would be valuable if future phylogenetic studies could distinguish between autopolyploids and allopolyploids so that the question of genome doubling and genome merger can be disentangled. Integrative approaches that combine molecular and ecophysiological tools that compare responses of polyploids grown in controlled conditions with those grown in natural environments might help in the future to get answers to some of the still unresolved questions, and help us better understand the ecological and evolutionary role that polyploidy has.

\section{DATA ARCHIVING}

There were no data to deposit.

\section{CONFLICT OF INTEREST}

The author declares no conflict of interest.

\section{ACKNOWLEDGEMENTS}

I thank Barbara Mable, Alexa Tullis, Cristy Walcher, members of my lab, and two anonymous reviewers for critical reading of the manuscript and suggestions for improvement. This article is based upon work in my lab and supported by the National Science Foundation under Grant No. 1118539 (to $\mathrm{AM}$ ).

Adams K, Wendel J (2005). Polyploidy and genome evolution in plants. Curr Opin Plant Biol 8: 135-141.

Adams KL, Cronn R, Percifield R, Wendel JF (2003). Genes duplicated by polyploidy show unequal contributions to the transcriptome and organ-specific reciprocal silencing. Proc Natl Acad Sci USA 100: 4649-4654.

Arrigo N, Barker MS (2012). Rarely successful polyploids and their legacy in plant genomes. Curr Opin Plant Biol 15: 140-146.

Bardil A, de Almeida JD, Combes MC, Lashermes P, Bertrand B (2011). Genomic expression dominance in the natural allopolyploid Coffea arabica is massively affected by growth temperature. New Phytol 192: 760-774.

Birchler JA, Yao H, Chudalayandi S, Vaiman D, Veitia RA (2010). Heterosis. The Plant Cell Online 22: 2105-2112.

Blanc G, Wolfe KH (2004). Widespread paleopolyploidy in model plant species inferred from age distributions of duplicate genes. Plant Cell 16: 1667-1678.

Buggs RJ, Elliott NM, Zhang L, Koh J, Viccini LF, Soltis DE et al. (2010). Tissue-specific silencing of homoeologs in natural populations of the recent allopolyploid Tragopogon mirus. New Phytol 186: 175-183.

Buggs RJA, Zhang L, Miles N, Tate JA, Gao L, Wei W et al. (2011). Transcriptomic shock generates evolutionary novelty in a newly formed, natural allopolyploid plant. Curr Biol 21: 551-556.

Chaudhary B, Flagel L, Stupar RM, Udall JA, Verma N, Springer NM et al. (2009). Reciprocal silencing, transcriptional bias and functional divergence of homeologs in polyploid cotton (gossypium). Genetics 182: 503-517.

Chelaifa H, Monnier A, Ainouche M (2010). Transcriptomic changes following recent natural hybridization and allopolyploidy in the salt marsh species Spartina $\times$ townsendii and Spartina anglica (Poaceae). New Phytol 186: 161-174.

Chen Z (2007). Genetic and epigenetic mechanisms for gene expression and phenotypic variation in plant polyploids. Annu Rev Plant Biol 58: 377-406.

Chen ZJ (2010). Molecular mechanisms of polyploidy and hybrid vigor. Trends Plant Sci 15: $57-71$.

Comai $L$ (2005). The advantages and disadvantages of being polyploid. Nat Rev Genet 6 : 836-846.

Comai L, Tyagi AP, Winter K, Holmes-Davis R, Reynolds SH, Stevens Y et al. (2000). Phenotypic instability and rapid gene silencing in newly formed Arabidopsis allotetraploids. Plant Cell 12: 1551-1568.

Combes M-C, Cenci A, HIn Baraille, Bertrand B/E, Lashermes P (2012). Homeologous gene expression in response to growing temperature in a recent allopolyploid (Coffea arabica L.). J Hered 103: 36-46.

Coyne J, Orr H (2004). Speciation. Sinauer Associates, Inc: Sunderland, MA.

Dong S, Adams KL (2011). Differential contributions to the transcriptome of duplicated genes in response to abiotic stresses in natural and synthetic polyploids. New Phytol 190: 1045-1057.

Doyle JJ, Doyle JL, Brown AHD (1999). Origins, colonization, and lineage recombination in a widespread perennial soybean polyploid complex. Proc Natl Acad Sci 96: 10741-10745.

Doyle JJ, Doyle JL, Rauscher JT, Brown AHD (2004). Evolution of the perennial soybean polyploid complex (Glycine subgenus Glycine): a study of contrasts. Biol J Linnean Soc 82: 583-597.

Flagel L, Udall J, Nettleton D, Wendel J (2008). Duplicate gene expression in allopolyploid Gossypium reveals two temporally distinct modes of expression evolution. BMC Biol 6: 16

Fryxell PA (1979). The Natural History of the Cotton Tribe. Texas A\&M University Press: College Station, TX, USA. 
Gaeta RT, Pires JC, Iniguez-Luy F, Leon E, Osborn TC (2007). Genomic changes in resynthesized Brassica napus and their effect on gene expression and phenotype. Plant Cell 19: 3403-3417.

Gaeta RT, Yoo S-Y, Pires JC, Doerge RW, Chen ZJ, Osborn TC (2009). Analysis of gene expression in resynthesized Brassica napus allopolyploids using arabidopsis 70mer oligo microarrays. PLOS ONE 4: e4760.

Grant V (1971). Plant Speciation, 1st edn. Columbia University Press: New York.

Grant V (1975). Genetics of flowering plants. Columbia University Press: New York.

Gu Z, Steinmetz LM, Gu X, Scharfe C, Davis RW, Li W-H (2003). Role of duplicate genes in genetic robustness against null mutations. Nature 421: 63-66.

Gustavsson A (1946). Apomixis in higher plants. Lunds Universitets Arsskrift 42-43: $1-370$.

Hagerup O (1932). Über polyploidie in beziehung zu klima, ökologie und phylogenie. Hereditas 16: 19-40.

Hanelt P (1966). Polyploidie-frequenz und geographische verbreitung bei höheren pflanzen. Biologische Rundschau 4: 183-196.

Hegarty M, Barker G, Wilson I, Abbott R, Edwards K, Hiscock S (2006). Transcriptome shock after interspecific hybridization in Senecio is ameliorated by genome duplication. Curr Biol 16: 1652-1659.

Hegarty MJ, Batstone TOM, Barker GL, Edwards KJ, Abbott RJ, Hiscock SJ (2011). Nonadditive changes to cytosine methylation as a consequence of hybridization and genome duplication in Senecio (Asteraceae). Mol Ecol 20: 105-113.

Hegarty MJ, Jones JM, Wilson ID, Barker GL, Coghill JA, Sanchez-Baracaldo P et al. (2005). Development of anonymous cDNA microarrays to study changes to the Senecio floral transcriptome during hybrid speciation. Mol Ecol 14: 2493-2510.

Hodgson JG, Sharafi M, Jalili A, Diaz S, Montserrat-Marti G, Palmer C et al. (2010). Stomatal vs. genome size in angiosperms: the somatic tail wagging the genomic dog? Ann Bot (Lond) 105: 573-584.

Jiao Y, Wickett NJ, Ayyampalayam S, Chanderbali AS, Landherr L, Ralph PE et al. (2011). Ancestral polyploidy in seed plants and angiosperms. Nature 473: 97-100.

Johnson HB (1975). Plant pubescence: an ecological perspective. Bot Rev 41: 233-258.

Kim E-D, Chen ZJ (2011). Unstable transcripts in arabidopsis allotetraploids are associated with nonadditive gene expression in response to abiotic and biotic stresses. PLOS ONE 6: e24251.

Lee HS, Chen ZJ (2001). Protein-coding genes are epigenetically regulated in Arabidopsis polyploids. Proc Natl Acad Sci USA 98: 6753-6758.

Levin D (1975). Minority cytotype exclusion in local plant populations. Taxon 24: 35-43.

Levin D (2002). The Role of Chromosomal Change in Plant Evolution. Oxford University Press: New York.

Lewis W (1980). Polyploidy in species populations. In: Lewis W (ed.). Polyploidy: Biological Relevance. Plenum: New York, pp 103-144.

Li X, Yu E, Fan C, Zhang C, Fu T, Zhou Y (2012). Developmental, cytological and transcriptional analysis of autotetraploid Arabidopsis. Planta 236: 579-596.

Li W-L, Berlyn GP, Ashton PMS (1996). Polyploids and their structural and physiologica characteristics relative to water deficit in Betula papyrifera (Betulaceae). Am J Bot 83 15-20.

Liu Z, Adams KL (2007). Expression partitioning between genes duplicated by polyploidy under abiotic stress and during organ development. Curr Biol 17: 1669-1674.

Lynch M (2007). The Origins of Genome Architecture. Sinauer: Sunderland, MA

Löve A (1953). Subarctic polyploidy. Hereditas 39: 113-124.

Löve A, Löve D (1949). The geobotanical significance of polyploidy. Portugaliae Acta (Suppl) 273-352.

Madlung A, Henkhaus N, Jurevic L, Kahsai EA, Bernhard J (2012). Natural variation and persistent developmental instabilities in geographically diverse accessions of the allopolyploid Arabidopsis suecica. Physiol Plant 144: 123-133.

Madlung A, Tyagi AP, Watson B, Jiang H, Kagochi T, Doerge RW et al. (2005). Genomic changes in synthetic Arabidopsis polyploids. Plant J 41: 221-230.

Maherali H, Walden AE, Husband BC (2009). Genome duplication and the evolution of physiological responses to water stress. New Phytol 184: 721-731.

Masterson J (1994). Stomatal size in fossil plants: evidence for polyploidy in majority of angiosperms. Science 264: 421-424.

Matsushita SC, Tyagi AP, Thornton GM, Pires JC, Madlung A (2012). Allopolyploidization lays the foundation for evolution of distinct populations: evidence from analysis of synthetic arabidopsis allohexaploids. Genetics 191: 535-547.

Mayfield D, Chen ZJ, Pires JC (2011). Epigenetic regulation of flowering time in polyploids. Curr Opin Plant Biol 14: 174-178.
Mayr E (1963). Animal Species and Evolution. Harvard University Press: Cambridge. Mayrose I, Zhan SH, Rothfels CJ, Magnuson-Ford K, Barker MS, Rieseberg LH et al. (2011). Recently formed polyploid plants diversify at lower rates. Science 333. 1257.

Melaragno JE, Mehrotra B, Coleman AW (1993). Relationship between endopolyploidy and cell size in epidermal tissue of arabidopsis. The Plant Cell Online 5: 1661-1668.

Moore RC, Purugganan MD (2005). The evolutionary dynamics of plant duplicate genes. Curr Opin Plant Biol 8: 122-128.

Müntzing A (1936). The evolutionary significance of autopolyploidy. Hereditas 21: 363-378.

Ohno S (1970). Evolution by Gene Duplication. Springer Verlag: New York.

Otto SP (2007). The evolutionary consequences of polyploidy. Cell 131: 452-462.

Pignatta D, Dilkes BP, Yoo SY, Henry IM, Madlung A, Doerge RW et al. (2010). Differential sensitivity of the Arabidopsis thaliana transcriptome and enhancers to the effects of genome doubling. New Phytol 186: 194-206.

Pumphrey M, Bai J, Laudencia-Chingcuanco D, Anderson O, Gill BS (2009). Nonadditive expression of homoeologous genes is established upon polyploidization in hexaploid wheat. Genetics 181: 1147-1157.

Ramsey J (2011). Polyploidy and ecological adaptation in wild yarrow. Proc Nat/ Acad Sci 108: 7096-7101.

Ramsey J, Schemske DW (1998). Pathways, mechanisms, and rates of polyploid formation in flowering plants. Annu Rev Ecol Syst 29: 467-501.

Rapp R, Udall J, Wendel J (2009). Genomic expression dominance in allopolyploids. BMC Biol 7: 18.

Rhoades MM, Dempsey E (1966). Induction of chromosome doubling at meiosis by the elongate gene in maize. Genetics 54: 505-522.

Riddle N, Jiang H, An L, Doerge R, Birchler J (2010). Gene expression analysis at the intersection of ploidy and hybridity in maize. TAG Theor App/ Genet 120: 341-353.

Riddle NC, Kato A, Birchler J (2006). Genetic variation for the response to ploidy change in Zea mays L. Theor App/ Genet 114: 101-111.

Schlueter JA, Dixon P, Granger C, Grant D, Clark L, Doyle JJ et al. (2004). Mining EST databases to resolve evolutionary events in major crop species. Genome 47: 868-876.

Soltis DE, Buggs RJA, Doyle JJ, Soltis PS (2010). What we still don't know about polyploidy. Taxon 59: 1387-1403.

Soltis P, Soltis D (2009). The role of hybridization in plant speciation. Ann Rev Plant Biol 60: 561-588.

Speckman G, Post J, Dijkstra H (1965). Length of stomata as an indicator for polyploidy in rye-grasses. Euphytica 14: 225-228.

Stebbins GJ (1950). Variation and Evolution in Plants. Columbia University Press: New York.

Stebbins GL (1947). Types of polyploids: their classification and significance. Adv Genet 1: 403-429.

Stupar RM, Bhaskar PB, Yandell BS, Rensink WA, Hart AL, Ouyang S et al. (2007). Phenotypic and transcriptomic changes associated with potato autopolyploidization. Genetics 176: 2055-2067.

Wang J, Tian L, Lee HS, Wei NE, Jiang H, Watson B et al. (2006). Genomewide nonadditive gene regulation in Arabidopsis allotetraploids. Genetics 172: 507-517.

Wang J, Tian L, Madlung A, Lee HS, Chen M, Lee JJ et al. (2004). Stochastic and epigenetic changes of gene expression in Arabidopsis polyploids. Genetics 167: 1961-1973.

Wendel JF, Cronn RC (2003). Polyploidy and the evolutionary history of cotton. Adv Agron 78: $139-186$

Winge 0 (1917). The chromosomes, their number and general importance. Compt Rend Trav Lab Carlsberg 13: 131-275.

Wood T, Takebayashi N, Barker M, Mayrose I, Greenspoon P, Rieseberg L (2009). The frequency of polyploid speciation in vascular plants. Proc Natl Acad Sci USA 106: $13875-13879$.

Wright KM, Pires JC, Madlung A (2009). Mitotic instability in resynthesized and natural polyploids of the genus Arabidopsis (Brassicaceae). Am J Bot 96: 1656-1664.

Xiong Z, Gaeta RT, Pires JC (2011). Homoeologous shuffling and chromosome compensation maintain genome balance in resynthesized allopolyploid Brassica napus. Proc Natl Acad Sci USA 108: 7908-7913.

Yu Z, Haberer G, Matthes M, Rattei T, Mayer KFX, Gierl A et al. (2010). Impact of natural genetic variation on the transcriptome of autotetraploid Arabidopsis thaliana. Proc Nat Acad Sci 107: 17809-17814. 